\title{
Clinical characteristics of hepatocellular carcinoma in elderly patients
}

\author{
TAKUYA HONDA, HISAMITSU MIYAAKI, TATSUKI ICHIKAWA, NAOTA TAURA, SATOSHI MIUMA, \\ HIDETAKA SHIBATA, HAJIME ISOMOTO, FUMINAO TAKESHIMA and KAZUHIKO NAKAO
}

\begin{abstract}
Department of Gastroenterology and Hepatology, Graduate School of Biomedical Sciences,
\end{abstract}
Nagasaki University, Nagasaki 852-8501, Japan

Received May 3, 2011; Accepted June 3, 2011

DOI: $10.3892 / 01.2011 .359$

\begin{abstract}
The incidence of hepatocellular carcinoma (HCC) in elderly patients in Japan has been on the increase. The aim of the present study was to evaluate the impact of aging on the clinicopathological findings and the survival of HCC patients. A total of 624 patients with HCC were examined in this study. The patients were classified according to their age at the time of diagnosis: one group comprised younger patients $(<75$ years; $n=544)$ and the second comprised elderly patients $[(\geq 75$ years; $n=80,(12 \%)]$. Results showed that there were significantly more female patients (younger:elderly, 22:36; $\mathrm{p}=0.005$ ), normal livers (younger:elderly, 0.3:6\%; $\mathrm{p}=0.0002$ ), non-viral HCC (younger:elderly, 11:31\%; p<0.001) and solitary tumors (younger:elderly, $53: 76 \% ; \mathrm{p}=0.0008$ ) in the elderly group. Five out of seven (71\%) non-B non-C (NBNC) HCC patients who developed HCC in the normal liver were elderly patients. Survival between the younger and elderly HCC groups was not significantly different (younger:elderly, 4.38:3.45 years; $\mathrm{p}=0.665$ ). Additionally, elderly HCC patients had fewer tumors, more mild underlying liver damage, and more frequent NBNC HCC. Their prognosis was not necessarily poorer than that of the younger HCC patients. Additionally, it appears that elderly patients develop HCC even without fibrosis. Therefore, aging may be a factor affecting hepatocarcinogenesis.
\end{abstract}

\section{Introduction}

Hepatocellular carcinoma (HCC) is one of the most common cancers $(1,2)$, with an estimated half a million cases annually, worldwide. Although HCC is generally diagnosed in middle-

Correspondence to: Dr Hisamitsu Miyaaki, Department of Gastroenterology and Hepatology, Graduate School of Biomedical Sciences, Nagasaki University, 1-7-1 Sakamoto, Nagasaki 852-8501, Japan

E-mail: miyaaki-hi@umin.ac.jp

Key words: hepatocellular carcinoma, aging, non-viral hepatocellular carcinoma aged and elderly individuals, the age distribution of HCC varies according to etiology. The differences in age at the time of diagnosis of HCC affect the treatment strategy.

The Japanese population has one of the longest average life spans, and the size of the aged population has been increasing rapidly. As a result, the prevalence of elderly patients with HCC has increased (3-5). There is some controversy regarding whether aging plays a role in the factors and survival of patients with HCC. Previous studies reported that the longterm survival of younger HCC patients is similar to that of elderly patients $(6,7)$. On the other hand, it has been reported that elderly HCC patients tended to have a poorer prognosis (8).

A recent increase in the number of elderly HCC patients in Japan has been reported $(4,9,10)$. However, the impact of aging on the emergence of HCC has yet to be adequately investigated. Therefore, the aim of the present study was to investigate the effect of aging on the clinicopathological findings and the survival of HCC patients.

\section{Patients and methods}

Patients. A total of 624 patients presenting with HCC at the Department of Gastroenterology and Hepatology, Nagasaki University School of Medicine, Japan, were recruited for this study, between October 1981 and October 2007. The diagnosis of HCC was based on $\alpha$-fetoprotein (AFP) levels, des- $\gamma$ carboxy prothrombin (DCP) levels, imaging studies including ultrasonography (USG), computerized tomography (CT), magnetic resonance imaging (MRI), hepatic angiography (HAG) and/or liver biopsy. The diagnosis of chronic liver disease and liver cirrhosis was based on the level of platelets and imaging studies and/or liver histology. The patients were classified into two groups according to their age at the time of diagnosis: a younger group ( $<75$ years; $n=544)$ and an elderly group ( $\geq 75$ years; $n=80$ ).

Etiology of HCC. A diagnosis of chronic hepatitis $\mathrm{C}$ virus (HCV) infection was based on the presence of $\mathrm{HCV}$ antibodies (microparticle enzyme immunoassay; Abbott Laboratories, Tokyo, Japan) and HCV-RNA detected by polymerase chain reaction (PCR), whereas the diagnosis of chronic HBV infection was based on the presence of hepatitis B surface antigen (HBs/Ag) (enzyme-linked immunosorbent assay; Abbot 
Table I. Patient characteristics.

Characteristics

\begin{tabular}{lc}
\hline Age (years) & $63.9 \pm 9.8$ \\
Gender, male : female & $478: 146$ \\
BMI & $22.6 \pm 3.2$ \\
Normal : CH : LC & $7: 120: 497$ \\
Child-Pugh grade & $6.3 \pm 1.6$ \\
NBNC : HBV : HCV & $74: 139: 430: 19$ \\
Tumor diameter $(\mathrm{cm})$ & $4.3 \pm 3.5$ \\
No. of tumors & $2.8 \pm 3.1$ \\
\hline
\end{tabular}

BMI, body mass index; $\mathrm{CH}$, chronic hepatitis; $\mathrm{LC}$, liver cirrhosis, $\mathrm{NBNC}$, non-B non-C; $\mathrm{HBV}$, hepatitis B virus; $\mathrm{HCV}$, hepatitis $\mathrm{C}$ virus .

Table II. Comparison of the patient backgrounds.

\begin{tabular}{lccc}
\hline & $<75$ Years old $\geq 75$ Years old & p-value \\
\cline { 2 - 4 } & 544 Cases & 80 Cases & \\
\hline Gender (female) & $117(22 \%)$ & $29(36 \%)$ & 0.0050 \\
Normal liver & $2(0.3 \%)$ & $5(6 \%)$ & 0.0002 \\
Liver cirrhosis & $440(80 \%)$ & $57(71 \%)$ & 0.0450 \\
Child-Pugh grade & $6.3 \pm 1.7$ & $6.0 \pm 2.2$ & 0.1650 \\
Prothrombin time $(\%)$ & $77 \pm 19$ & $79 \pm 24$ & 0.4600 \\
Bilirubin $(\mathrm{mg} / \mathrm{dl})$ & $1.5 \pm 2.4$ & $1.0 \pm 0.7$ & 0.1080 \\
Albumin $(\mathrm{g} / \mathrm{dl})$ & $3.8 \pm 3.2$ & $3.6 \pm 0.5$ & 0.7380 \\
\hline
\end{tabular}

Table III. Comparison of risk factors for hepatocellular carcinoma.

\begin{tabular}{lrrr}
\hline & $<75$ Years old & $\geq 75$ Years old & p-value \\
\cline { 2 - 4 } & 544 Cases & 80 Cases & \\
\hline HBsAg-positive & $131(24 \%)$ & $8(10 \%)$ & 0.004 \\
HCVAb-positive & $381(70 \%)$ & $49(61 \%)$ & 0.112 \\
NBNC & $59(11 \%)$ & $25(31 \%)$ & 0.001 \\
Diabetes mellitus & $152(28 \%)$ & $22(28 \%)$ & 0.934 \\
Alcohol consumption & $117(22 \%)$ & $10(12 \%)$ & 0.085 \\
\hline
\end{tabular}

HBsAg, hepatitis B surface antigen; HCVAb, Hepatitis C antibody; NBNC, non-B non-C.

Laboratories). The history of alcohol intake was noted from medical records. Habitual drinking was defined as an average daily consumption of an amount equivalent to $80 \mathrm{~g}$ of pure ethanol over a period of $>10$ years.

Statistical analysis. The SPSS 9.0 for Windows statistical software program was used to assess correlations among multiple variables. When appropriate, clinical and laboratory
Table IV. Comparison of tumor characteristics and therapy for hepatocellular carcinoma.

\begin{tabular}{lccc}
\hline & $<75$ Years old & $\geq 75$ Years old & p-value \\
\cline { 2 - 4 } & 544 Cases & 80 Cases & \\
\hline Diameter $(\mathrm{cm})$ & $4.2 \pm 3.4$ & $4.3 \pm 3.9$ & 0.8250 \\
No. of tumors & $4.4 \pm 5.2$ & $1.9 \pm 2.3$ & 0.0060 \\
Solitary cases & $293(53 \%)$ & $56(76 \%)$ & 0.0008 \\
TNM, stage I or II & $338(62 \%)$ & $59(73 \%)$ & 0.0430 \\
Surgical resection & $68(12.5 \%)$ & $7(9 \%)$ & 0.3350 \\
Local ablative therapy & $144(26 \%)$ & $27(33 \%)$ & 0.1780 \\
TACE & $260(47 \%)$ & $40(50 \%)$ & 0.7130 \\
\hline
\end{tabular}

TACE, transarterial chemoembolization.

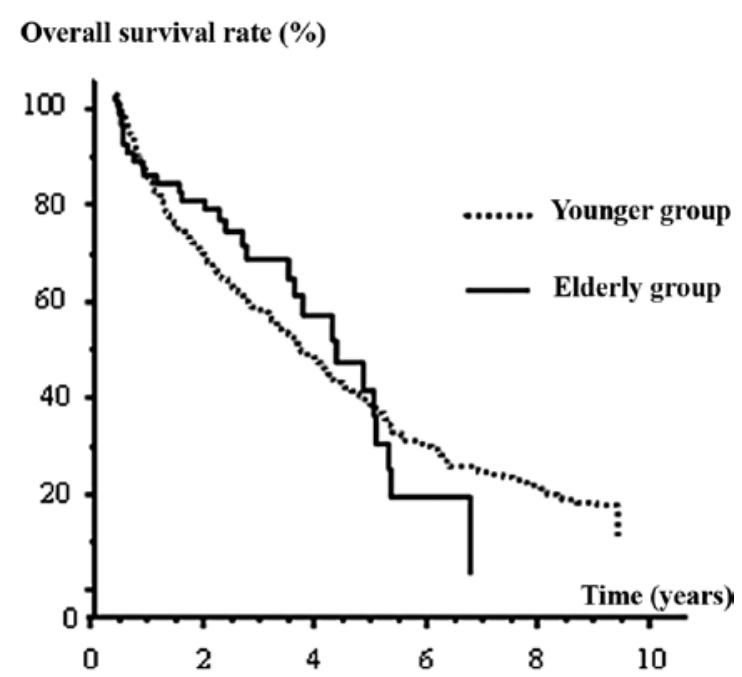

Figure 1. Kaplan-Meier model of the overall survival rate for the younger and elderly groups (younger HCC, 544 cases; elderly HCC, 80 cases). The overall survival between the younger and elderly HCC groups was not significantly different $(\mathrm{p}=0.665)$.

data were compared with a $\chi^{2}$ analysis, the Student's t-test or the Mann-Whitney test. The survival from time of diagnosis of HCC was analyzed using the Kaplan-Meier method and compared using the log-rank method. $\mathrm{P}<0.05$ was considered to be statistically significant.

\section{Results}

Of the 624 patients, 80 (12\%) patients were aged 75 years or older. The mean age of these older patients was 78.7 \pm 3.6 . The clinical characteristics of the patients are shown in Table I. Significantly more patients in the elderly group were female $(22: 36 \% ; p=0.005)$. The incidence of patients with liver cirrhosis was significantly higher, and the presence of a normal liver was significantly lower in the younger group than in the elderly group $(80: 71 \% ; \mathrm{p}=0.045 ; 0.3: 6 \%, \mathrm{p}=0.0002)$. No significant differences were observed in the protrobin time, total bilirubin, albumin or liver function as expressed by the Child-Pugh grade between the two groups (Table II). 
Regarding viral status, the number of patients positive for HBsAg was significantly lower in the elderly group (24:10\%, $\mathrm{p}=0.004)$, and the number of patients who were HBsAg and $\mathrm{HCV}$ antibody-negative [non-B non-C (NBNC)] was higher in the elderly group than in the younger group $(11: 31 \%, \mathrm{p}=0.001)$.

In the NBNC HCC patients in the elderly group, 6 of 23 patients showed chronic hepatitis and 5 of 25 showed normal livers. No significant differences were found between the younger and elderly HCC groups with regards to alcoholism and diabetes mellitus (Table III).

No significant differences were noted in the tumor diameter between the younger and elderly groups. The number of HCC nodules was significantly lower in the elderly group than that in the younger group $(4.4 \pm 5.2: 1.9 \pm 2.3, p=0.006)$. The incidence of solitary cases and TNM stage I or II disease was significantly higher in the elderly group compared to that of the younger group $(53: 76 \%, \mathrm{p}=0.0008 ; 62: 73 \%, \mathrm{p}=0.043)$. No significant differences were found between the younger and elderly HCC groups with regards to surgery, ablation therapy and transarterial chemoembolization (TACE) (Table IV).

The overall survival rate between the younger and elderly HCC groups was not significantly different $(p=0.665)$. The overall median survival for the younger group was 4.38 years, compared with 3.45 years for the elderly group (Fig. 1).

\section{Discussion}

Age at diagnosis has been shown to have significant prognostic value in certain types of cancer. Although the number of elderly patients with HCC is on the increase in Japan $(3,4)$, the characteristics and prognosis of HCC in elderly patients has yet to be elucidated. In this study, patients with HCC aged 75 years or older were examined, and their clinicopathological characteristics were identified and compared to those of the younger patients.

There were more male patients presenting with HCC in the younger group as compared to the elderly patients; one of the reasons for this being the difference in viral status. In this study, HBV infection, which is more common in males $(11,12)$, was lower in the elderly group than in the younger group. Moreover, males were more likely to be heavy drinkers.

The prevalence of a normal liver was higher, whereas that of liver cirrhosis was lower in the elderly group. Of note is that 5 of $23(21 \%)$ patients with NBNC HCC in the elderly group had normal livers. Additionally, 5 of 7 patients whose HCC developed in a normal liver were in the elderly group.

Chronic inflammation and viral infection are considered to be significant risk factors for $\mathrm{HCC}$, but the elderly patients recruited in this study had neither factor. A previous study reported that the telomere length in the liver is shortened, not only with the progression of fibrosis staging, but also with aging (13). Moreover, the reduction of telomere length has been reported to increase the risk of HCC (14). Thus, elderly patients may have shorter telomeres, predisposing them to develop HCC, even if chronic liver disease was not prevalent. Findings of various studies have suggested that aberrant DNA methylation is a crucial epigenetic alteration in HCC (15-17). Some of the aberrant methylation observed in human cancer may be a consequence of chronic viral inflammation $(18,19)$. On the other hand, aberrant methylation is also observed in the normal aging process (20), and may contribute to the occurrence of HCC in elderly patients with normal livers.

In this study, the HBV infection rate was lower, while the NBNC rate was higher in the elderly group than that in the younger group. Previous reports have shown that the average age of diagnosis of HBV-related $\mathrm{HCC}$ is approximately 55 years of age, whereas that of HCV-related HCC is approximately 65 years of age, and that of NBNC HCC is approximately 70 years of age $(3,4)$. In Japan, the predominant time of transmission of the hepatitis B virus is during the prenatal period. The subsequent genomic long interreactions from an early age may lead to hepatocarcinogenesis at a younger age in the infected individuals.

On the other hand, patients with non-alcoholic steatohepatitis (NASH)-related HCC are older at diagnosis than those with HCC related to HBV and HCV $(21,22)$. These results suggest that some of the NBNC HCC are NASH-related HCC.

The number of HCC nodules was lower, and the prevalence of single nodule $\mathrm{HCC}$ was higher in the elderly group than that in the younger group. Two main types of HCC occurrence exist, the first of which occurs at the time of the initial diagnosis with multicenter occurrence, which is associated with the degree of underlying liver damage. In this study, the prevalence of liver cirrhosis in the elderly group was lower than that in the younger group. The mild underlying liver damage in the elderly group may be associated with the smaller number of tumors observed in these patients.

Since elderly patients had fewer tumors and milder underlying liver damage at the time of the initial diagnosis, a more favorable prognosis in the elderly group may be expected. In this study, the overall survival rate was not significantly different between the two HCC groups. Overall, the majority of the elderly patients experienced various comorbidities, including cardiovascular disease, respiratory disease and diabetes mellitus. Taken together, the causes of death unrelated to HCC may have affected the survival rate in the elderly group.

In conclusion, elderly HCC patients had fewer tumors, milder underlying liver damage, and more frequent NBNC HCC. Additionally, it appears that elderly patients develop HCC even without fibrosis. Aging may therefore be a factor affecting hepatocarcinogenesis.

\section{References}

1. Bosch FX, Ribes J, Diaz M and Cleries R: Primary liver cancer: worldwide incidence and trends. Gastroenterology 127: S5-S16, 2004.

2. El-Serag HB and Mason AC: Risk factors for the rising rates of primary liver cancer in the United States. Arch Intern Med 160: 3227-3230, 2000.

3. Kiyosawa K, Umemura T and Ichijo T, et al: Hepatocellular carcinoma: recent trends in Japan. Gastroenterology 127: S17-S26, 2004.

4. Taura N, Hamasaki K, Nakao K, et al: Aging of patients with hepatitis $\mathrm{C}$ virus-associated hepatocellular carcinoma: long-term trends in Japan. Oncol Rep 16: 837-843, 2006.

5. Kiyosawa K and Tanaka E: Characteristics of hepatocellular carcinoma in Japan. Oncology 62 (Suppl 1): 5-7, 2002.

6. Ng IO, Ng MM, Lai EC and Fan ST: Pathologic features and patient survival in hepatocellular carcinoma in relation to age. J Surg Oncol 61: 134-137, 1996.

7. Lam CM, Chan AO, Ho P, et al: Different presentation of hepatitis B-related hepatocellular carcinoma in a cohort of 1863 young and old patients - implications for screening. Aliment Pharmacol Ther 19: 771-777, 2004. 
8. Falkson G, Cnaan A, Schutt AJ, Ryan LM and Falkson HC: Prognostic factors for survival in hepatocellular carcinoma Cancer Res 48: 7314-7318, 1988

9. Ohishi W, Kitamoto M, Aikata $\mathrm{H}$, et al: Impact of aging on the development of hepatocellular carcinoma in patients with hepatitis C virus infection in Japan. Scand J Gastroenterol 38 : 894-900, 2003.

10. Hamada H, Yatsuhashi H, Yano K, et al: Impact of aging on the development of hepatocellular carcinoma in patients with posttransfusion chronic hepatitis C. Cancer 95: 331-339, 2002.

11. Chen CJ, Yang HI, Su J, et al: Risk of hepatocellular carcinoma across a biological gradient of serum hepatitis B virus DNA level. Jama 295: 65-73, 2006.

12. Chen CL, Yang HI, Yang WS, et al: Metabolic factors and risk of hepatocellular carcinoma by chronic hepatitis B/C infection: a follow-up study in Taiwan. Gastroenterology 135: 111-121, 2008.

13. Aikata H, Takaishi H, Kawakami Y, et al: Telomere reduction in human liver tissues with age and chronic inflammation. Exp Cell Res 256: 578-582, 2000.

14. Isokawa O, Suda T, Aoyagi Y, et al: Reduction of telomeric repeats as a possible predictor for development of hepatocellular carcinoma: convenient evaluation by slot-blot analysis Hepatology 30: 408-412, 1999.

15. Yu J, Ni M, Xu J, et al: Methylation profiling of twenty promoter$\mathrm{CpG}$ islands of genes which may contribute to hepatocellular carcinogenesis. BMC Cancer 2: 29, 2002.
16. Lee S, Lee HJ, Kim JH, Lee HS, Jang JJ and Kang GH: Aberrant $\mathrm{CpG}$ island hypermethylation along multistep hepatocarcinogenesis. Am J Pathol 163: 1371-1378, 2003.

17. Yang B, Guo M, Herman JG and Clark DP: Aberrant promoter methylation profiles of tumor suppressor genes in hepatocellular carcinoma. Am J Pathol 163: 1101-1107, 2003.

18. Ushijima T: Detection and interpretation of altered methylation patterns in cancer cells. Nat Rev Cancer 5: 223-231, 2005.

19. Issa JP, Ottaviano YL, Celano P, Hamilton SR, Davidson NE and Baylin SB: Methylation of the oestrogen receptor CpG island links ageing and neoplasia in human colon. Nat Genet 7: 536-540, 1994 .

20. Huja N, Li Q, Mohan AL, Baylin SB and Issa JP: Aging and DNA methylation in colorectal mucosa and cancer. Cancer Res 58: 5489-5494, 1998.

21. Hashimoto E, Yatsuji S, Tobari M, et al: Hepatocellular carcinoma in patients with nonalcoholic steatohepatitis. J Gastroenterol 44 (Suppl 19): 89-95, 2009

22. Ascha MS, Hanouneh IA, Lopez R, Tamimi TA, Feldstein AF and Zein NN: The incidence and risk factors of hepatocellular carcinoma in patients with nonalcoholic steatohepatitis. Hepatology 51: 1972-1978, 2010. 